\title{
Immunohistochemical Analysis of p16 Expression in Uterine Smooth Muscle Tumors
}

\author{
Seiji Kanayama ${ }^{1}$, Hidekazu 0i1 ${ }^{1}$ Ryuji Kawaguchi², Naoto Furukawa², \\ Hiroshi Kobayashi ${ }^{2}$ \\ ${ }^{1}$ Department of Obstetrics and Gynecology, Nara Hospital, Faculty of Medicine, Kinki University, Nara, Japan \\ ${ }^{2}$ Department of Obstetrics and Gynecology, Nara Medical University, Nara, Japan \\ Email: ${ }^{*}$ kanayama@nara.med.kindai.ac.jp
}

Received 1 September 2015; accepted 4 October 2015; published 7 October 2015

Copyright (C) 2015 by authors and Scientific Research Publishing Inc.

This work is licensed under the Creative Commons Attribution International License (CC BY). http://creativecommons.org/licenses/by/4.0/

(C) (1) Open Access

\begin{abstract}
Objective: Immunohistochemistry with p16 antibody is used as a diagnostic marker in several gynecologic pathologies. The purpose of the present study was to evaluate the diagnostic and prognostic significance of p16 immunohistochemistry in various uterine smooth muscle tumors. Methods: Tissue specimens, including 29 leiomyomas, 13 smooth muscle tumors of uncertain malignant potential (STUMP), and 17 leiomyosarcomas, were obtained from 59 patients with uterine smooth muscle tumors. The prevalence of p16 expression in various uterine smooth muscle tumors was examined using immunohistochemistry methods and we investigated the association between p16 expression and various clinicopathologic parameters, including prognosis of leiomyosarcomas. Results: The prevalence of p16 expression of leiomyomas, STUMP, and leiomyosarcomas tumors was $10.3 \%, 38.4 \%$, and $76.4 \%$, respectively. The prevalence and immunohistochemistry scores of p16 were significantly higher in the leiomyosarcomas group than in the leiomyomas or STUMP tumor groups. In the leiomyosarcomas groups, p16 immunohistochemistry scores were significantly higher in the groups with more advanced stage, higher mitotic activity, and recurrence. The relationship among p16 immunohistochemistry scores and age, tumor size, extent of necrosis, and degree of myometrial invasion was not significant. High expression of p16 in leiomyosarcomas was linked to shorter disease-free and overall survival. Conclusions: The use of p16 immunohisitochemistry is a useful marker in differential diagnosis in various uterine smooth muscle tumors. Moreover, p16 overexpression possibly plays an important role in tumor progression and we demonstrate that $\mathrm{p} 16$ may be a predictor of overall survival in patients with leiomyosarcoma tumors.
\end{abstract}

\footnotetext{
"Corresponding author.
}

How to cite this paper: Kanayama, S., Oi, H., Kawaguchi, R., Furukawa, N. and Kobayashi, H. (2015) Immunohistochemical Analysis of p16 Expression in Uterine Smooth Muscle Tumors. Open Journal of Obstetrics and Gynecology, 5, 688-697. 


\section{Keywords}

\section{Leiomyoma, Leiomyosarcomas, p16, Smooth Muscle Tumors, STUMP}

\section{Introduction}

Smooth muscle tumors of the uterus are common. In general, they are classified as leiomyomas, smooth muscle tumors of uncertain malignant potential (STUMP), and leiomyosarcomas, and the majority of those tumors are benign leiomyomas. Uterine leiomyosarcomas are rare, accounting for only $1 \%$ of uterine cancers. Leiomyosarcomas are clinically very aggressive malignant smooth muscle tumors having a poor prognosis with cure rates ranging from $20 \%$ to $60 \%$, even when diagnosed at an early stage [1]. Therefore, differential diagnosis of malignant smooth muscle tumors from benign uterine smooth muscle tumors is a clinically serious problem. Pathologic diagnosis of leiomyosarcomas is not usually very difficult because leiomyosarcomas exhibit cell coagulative necrosis, high mitotic activity, and diffuse marked nuclear atypia. Diagnosis of leiomyosarcoma is limited, however, to only the morphologic features because these microscopic findings often overlap with those of STUMP. When the tumors lack more than two findings as above, these cases are diagnosed as variant-type leiomyoma or STUMP [2]. The etiology of leiomyosarcomas is unknown, but cell cycle regulators are thought to be intimately involved in the pathogenesis [3]. The loss of cell cycle control is a critical step in tumorigenesis. Several oncogenes and tumor suppressor genes involved in cell cycle control have been investigated and inactivation of retinoblastoma or $\mathrm{p} 16$, overexpression of $\mathrm{p} 53$, and $\mathrm{p} 21$ are reported to be closely involved in soft tissue sarcoma [4]-[6]. The p16 protein is a tumor-suppressor gene that acts as a negative cell cycle regulator [7]. p16 binds specifically to the cyclin-dependent kinase CDK-4, inhibiting the catalytic activity of the CDK4-cyclin D complex. Activation of p16 consequently blocks the transcription of cell-cycle regulatory proteins and results in cell-cycle arrest [8]. Therefore, loss of p16 functions, such as hypermethylation or deletion in promoter sequences, facilitates cell proliferation and is reported in many different types of carcinomas such as lung, breast, bladder, and ovarian carcinoma [9]-[14].

The detection of p16 protein using immunohistochemistry (IHC) methods has been applied for the differential diagnosis in the field of gynecologic pathology. Many studies have reported high p16 protein expression levels in precancerous or invasive lesions of the uterine cervix. Therefore, diffuse expression of p16 in the uterine cervix is regarded as an excellent surrogate marker of the presence of high-risk human papillomavirus (HPV) [15][17]. In addition, p16 is highly expressed in endometrial malignant tumors, especially serous adenocarcinoma and small cell carcinoma, compared with endometrioid adenocarcinoma [18]. Moreover, with respect to uterine smooth muscle tumors, several recent reports show frequent overexpression of p16 in uterine leiomyosarcoma compared with leiomyoma. These findings suggest that the detection of p16 expression may be a useful diagnostic marker of leiomyosarcoma [19]-[21]. The relationship between p16 expression and clinical parameters in smooth muscle tumors, however, has not been well examined. Therefore, in the present study, to assess the clinical significance of p16 expression, we examined the prevalence of $\mathrm{p} 16$ in various uterine smooth muscle tumors using IHC methods and analyzed the association with various clinicopathologic parameters, including prognosis.

\section{Materials and Methods}

The present study was a retrospective investigation of the relation between immunohisto-chemical p16 status and various clinicopathologic parameters, including prognosis in patients with uterine smooth muscle tumors. The present study was conducted in accordance with the principles of the Declaration of Helsinki. We retrospectively evaluated consecutive 59 patients diagnosed with uterine smooth muscle tumors, including 29 leiomyomas, 13 STUMPs, and 17 consecutive leiomyosarcomas. The study protocol was approved by the Institutional Review Board of Nara Medical University. Medical records and tissue specimens were obtained from 59 patients who had undergone hysterectomies or myomectomies between 1996 and 2013 at the Department of Gynecology of Nara Medical University. The 29 leiomyomas comprised usual types, cellular leiomyomas, bizarre types, mitotically-active types, and myxoid types (shown in Table 1). Histologic diagnoses of all cases were confirmed by review of hematoxylin and eosin-stained slides by two pathologists. Microscopic findings, such as mitotic activity, cellularity, necrosis, and nuclear atypia, were analyzed (shown in Table 2). Clinical patient character- 
Table 1. Summary of characteristics in 59 uterine smooth muscle tumors.

\begin{tabular}{lcc}
\hline \multicolumn{2}{c}{ Uterine smooth muscle tumors: total 59 } \\
& Diagnosis & Number \\
\hline Leiomyoma (LM) & 29 \\
Cullular & 7 \\
Bizarre & 2 \\
Mitotically active & 3 \\
Myxoid & 1 \\
$\begin{array}{l}\text { Uterine smooth muscle tumors of uncertain malignant } \\
\text { potential (STUMP) }\end{array}$ & 13 \\
Leiomyosarcoma (LMS) & 17 \\
\hline
\end{tabular}

Table 2. Clinicopathologic features of the 59 uterine smooth muscle tumors.

\begin{tabular}{lccc}
\hline & LM & STUMP & LMS \\
\hline \multicolumn{1}{c}{ N } & 29 & 13 & 17 \\
\hline Mean size (cm) & $8.1(10-18)$ & $10.5(3-19)$ & $11.2(3.5-18)$ \\
Nuclear atypia & & & \\
None or mild & 25 & 5 & 0 \\
Moderate-severe & 4 & 8 & 17 \\
Mitosis (/10 HPFs) & & & 5 \\
0 - 4 & 29 & 9 & 4 \\
5 - 10 & 3 & 4 & 8 \\
$>10$ & 0 & 0 & 1 \\
Necrosis & & & 13 \\
ITN & 2 & 5 & $2 / 17$ \\
CTCN & 2 & 7 & \\
LVSI & $0 / 29$ & $0 / 13$ & \\
\hline
\end{tabular}

ITN: infarct-type necrosis; CTCN: cagulative tumor cellnecrosis; LVSI: lymphovascular space involvement.

ristics are shown in Table 3. IHC for p16 was performed on representative 4- $\mu \mathrm{m}$ sections from formalin-fixed paraffin-embedded tissue sections using the BondMax automated immunostainer according to the standard protocol (p16 [INK4a] mouse monoclonal antibody; clone E6H4; dilution, 1:25; Dako). Cervical cancer tissue sections were used as a positive control, and omission of primary antibody was used as a negative control. The p16 IHC slides were evaluated using previously published criteria [15].

And semi-quantitatively scored according to the extent of stained nuclear or cytoplasmic cells as follows; 0 : no staining, $1+: 1 \%-10 \%$ of cells stained, $2+: 11 \%-25 \%, 3+: 26 \%-50 \%$, and $4+: 51 \%-100 \%$; negative means $0 \%$ area staining, focally positive means $1 \%-50 \%$ area staining, diffusely positive means $51 \%-100 \%$ area staining. All other staining patterns were scored as negative. Each lesion was examined and scored separately by two pathologists. The prevalence of p16 expression in various uterine smooth muscle tumors was examined and the association between p16 expression and various clinicopathologic parameters, including prognosis of leiomyosarcoma, was investigated. 
Table 3. Clinical patient characteristics of 59 uterine smooth muscle tumors.

\begin{tabular}{|c|c|c|c|}
\hline & LM & STUMP & LMS \\
\hline $\mathrm{N}$ & 29 & 13 & 17 \\
\hline Mean age & 45.3 & 44.7 & 56.1 \\
\hline (range) & $33-71$ & $35-63$ & $38-85$ \\
\hline BMI & $22.1 \pm 3.8$ & $22.1 \pm 2.6$ & $22.3 \pm 4.1$ \\
\hline \multicolumn{4}{|l|}{ Operation } \\
\hline Hysterectomy & 19 & 9 & 14 \\
\hline Myomectomy & 10 & 4 & 0 \\
\hline Biopsy & 0 & 0 & 2 \\
\hline \multicolumn{4}{|l|}{ Stage } \\
\hline I & & & 11 \\
\hline II & & & 1 \\
\hline III & & & 4 \\
\hline IV & & & 1 \\
\hline Recurrence & $0 / 29$ & $0 / 13$ & $7 / 17$ \\
\hline \multicolumn{4}{|l|}{ Prognosis } \\
\hline NED & 29 & 13 & 10 \\
\hline AWD & 0 & 0 & 1 \\
\hline DOD & 0 & 0 & 6 \\
\hline
\end{tabular}

NED: no evidence of disease; AWD: alive with disease; DOD: died of disease.

\section{Statistical Analysis}

Statistical analyses were performed using StatView software (SAS Institute Inc., Cary, NC, USA). Fisher exact test and Mann-Whitney U-test were used to analyze the differences in p16 expression positivity between leiomyoma, STUMP, and leiomyosarcoma. The differences between p16 expression scores and various clinicopathologic parameters in leiomyosarcoma were determined by the Mann-Whitney U-test. Kaplan-Meier method was used to calculate progression-free survival and overall survival rates, and differences in survival curves were evaluated with the log-rank test. $p$ values of less than 0.05 were considered statistically significant.

\section{Results}

IHC staining revealed the expression of p16 protein in both the nucleus and cytoplasm of the affected cells (Figure 1). The clinicopathologic features of uterine smooth muscle tumors are shown in Table 2. The prevalence of p16 expression of leiomyoma, STUMP, and leiomyosarcoma tumors was $10.3 \%, 38.4 \%$, and $76.4 \%$, respectively. The prevalence and p16 IHC scores were significantly higher in the leiomyosarcoma tumors compared with the leiomyoma and STUMP tumors $(\mathrm{p}<0.05$; Figure 2$)$. The prevalence and $\mathrm{p} 16$ IHC scores were significantly higher in the STUMP tumors than in the leiomyoma tumors $(\mathrm{p}<0.05$; Figure 2$)$. We also evaluated the p16 expression levels with the clinicopathologic features in leiomyosarcoma (Table 4). In patients with leiomyosarcoma, the p16 IHC score was significantly positively correlated with the clinical stage $(\mathrm{p}=$ $0.049)$, mitotic activity $(\mathrm{p}=0.0074)$, and relapse rate $(\mathrm{p}=0.0446)$. The $\mathrm{p} 16 \mathrm{IHC}$ scores did not correlate with age, tumor size, extent of necrosis, or degree of myometrial invasion. The progression-free survival and overall survival rates were significantly different between patients with tumors expressing high p16 levels and those with tumors expressing low p16 levels $(\mathrm{p}<0.05$; Figure 3 ). 


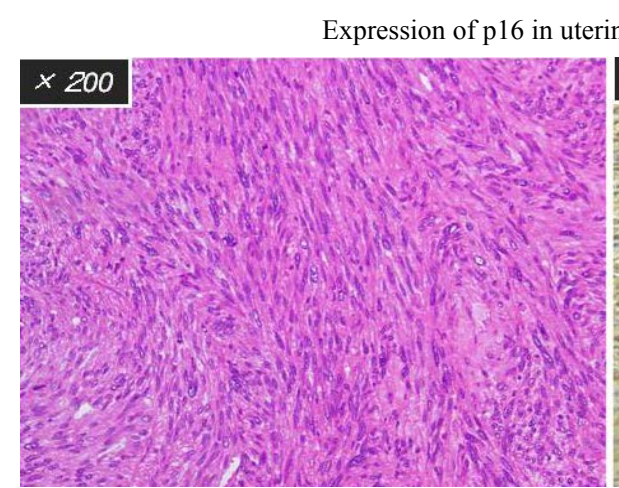

LMS HE staining

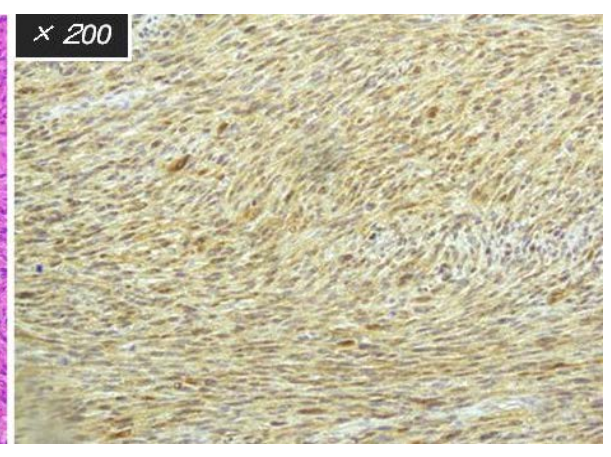

P16 expression in LMS

Figure 1. Representative p16 immunohistochemistry result showing 5+ staining in leiomyosarcoma. The p16 protein is expressed both within the nucleus and the cytoplasm of the tumor cells.

$\left.\left.\begin{array}{|lcc|}\hline \text { P16 positivity } & \% & \text { (n) } \\
\hline \text { LM } & 10.3 \\
\text { LM NOS } & 0.06 \\
\text { LMS variants } & 15.3 \\
& \\
\text { STUMP } & 38.4\end{array}\right] * \begin{array}{c}(3 / 29) \\
(1 / 16) \\
(2 / 13) \\
\\
\text { LMS }\end{array}\right]$\begin{tabular}{l}
$(5 / 13)$ \\
$(13 / 17)$ \\
\hline
\end{tabular}

$* \mathrm{p}<0.05$ by Fisher exact test

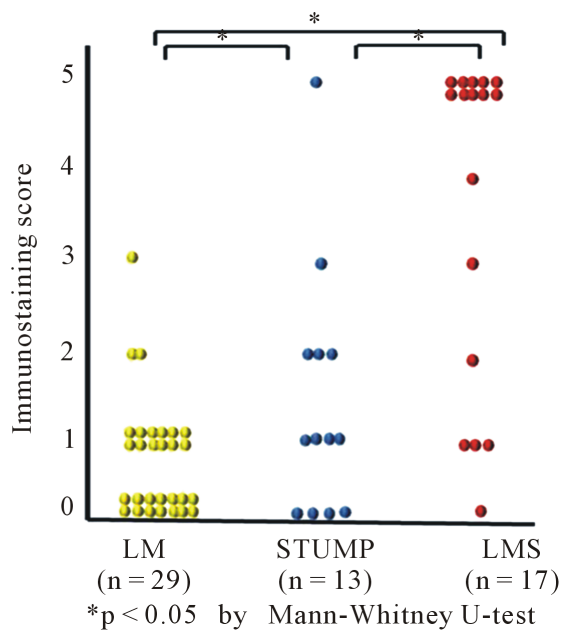

Figure 2. Prevalence and immunostaining scores of p16 expression in various uterine smooth muscle tumors.

Progression free and overall survival curves according to $\mathrm{p} 16$ expression

(a) PFS

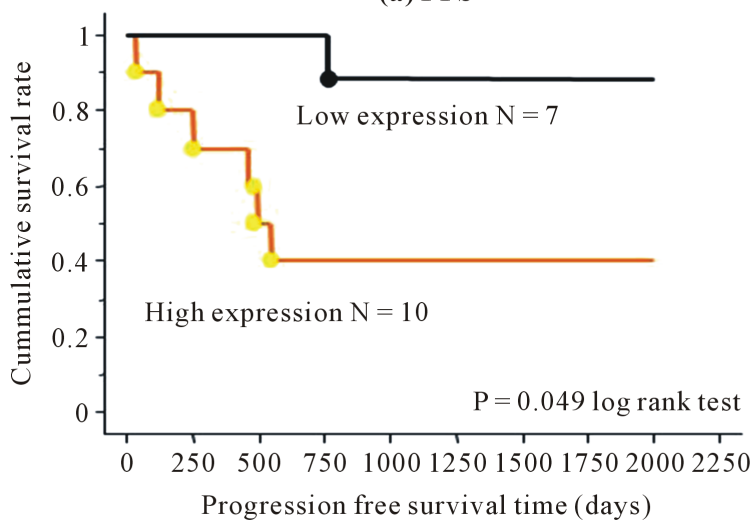

Kaplan-Meier (b) OS

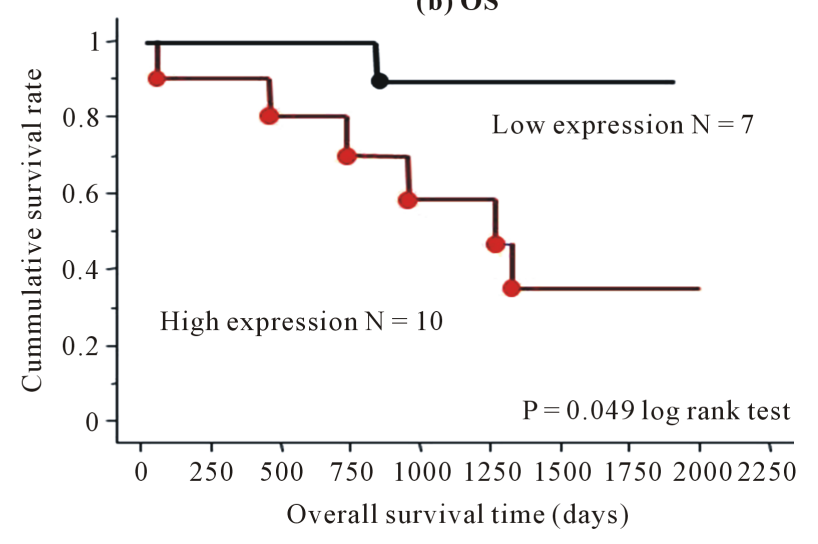

Kaplan-Meier

Figure 3. Survival outcomes for p16 high and low in leiomyosarcoma patients; (a) Progression-free survival (PFS) outcome for p16 high and low p16-expressing leiomyosarcomas; (b) Overall survival (OS) outcome for high and low p16-expressing leiomyosarcomas. 
Table 4. Correlation between p16 expression and clinicopathological parameters in leiomyosarcoma patients.

\begin{tabular}{|c|c|c|c|}
\hline \multicolumn{4}{|c|}{ Mann-Whitney's U test } \\
\hline Characteristic & Number & Staining score of p16 & $\mathrm{p}$ value \\
\hline \multicolumn{4}{|l|}{ Age (yrs) } \\
\hline$<50$ & 7 & 3.28 & \\
\hline$\geq 50$ & 10 & 3.9 & 0.524 \\
\hline \multicolumn{4}{|l|}{ Clinical stage (FIGO) } \\
\hline I & 11 & 3.0 & \\
\hline II-IV & 6 & 4.8 & 0.049 \\
\hline \multicolumn{4}{|l|}{ Tumor Size (cm) } \\
\hline$<10$ & 5 & 4.2 & \\
\hline$\geq 10$ & 12 & 3.4 & 0.449 \\
\hline \multicolumn{4}{|l|}{ Necrosis (\%) } \\
\hline$<30$ & 8 & 2.8 & \\
\hline$\geq 30$ & 9 & 4.3 & 0.110 \\
\hline \multicolumn{4}{|c|}{ Degress of myometrial invasion } \\
\hline Surface & 6 & 4.5 & \\
\hline Deep & 11 & 3.1 & 0.171 \\
\hline \multicolumn{4}{|c|}{ Mitosis (mean mitoses/10 HPF) } \\
\hline$<10$ & 10 & 2.7 & \\
\hline$\geq 10$ & 7 & 5.0 & 0.0074 \\
\hline \multicolumn{4}{|l|}{ Recurrence } \\
\hline No & 10 & 2 & \\
\hline Yes & 7 & 4.7 & 0.0446 \\
\hline
\end{tabular}

\section{Discussion}

The p16 (CDKN2a/INK4a) gene is located on the short arm of chromosome 9, region 9p21, and is a well-known tumor-suppressor gene. Loss of p16 function is reported in various malignant tumors, such as lung and ovary carcinoma [14] [22]-[24], and decreased p16 expression is associated with tumor progression and poor survival [23] [24]. Inactivation events are mainly due to homozygous deletion and methylation of the promoter, homozygous deletion, and point mutation, leading to intensified proliferation of neoplastic cells and cytostatic drug resistance. Frequent mutations and deletions of p16 are observed in many primary tumors and in human cancer cell lines [25]-[27]. Moreover, the loss of p16 may be an early event in cancer progression, because deletion of at least one copy is quite high in some premalignant lesions. In contrast, overexpression of p16 protein is reported in several malignant tumors [11] [28] [29]. In gynecologic tumors, p16 over-expression is well known in uterine cervical pre- and cancerous lesions. Diffuse positivity for p16 in the uterine cervix is considered a surrogate marker of the presence of active high-risk HPV [15]-[17]. HPV-associated tumors, other than uterine cervical tumors such as oropharynx and some head and neck cancers, are also usually positive for p16. The E7 protein is a major oncoprotein produced by infection with high-risk HPV and binds to retinoblastoma protein, resulting in its functional inactivation. It has been hypothesized that p16 is under negative feedback control of functional retinoblastoma, so the function of p16 is normal and overexpression of p16 is thought to occur in cells 
infected by high-risk HPV E7. Several reports have recently demonstrated overexpression of p16 protein in leiomyosarcomas compared with leiomyomas [19]-[21]. Why p16 protein, a tumor suppressor, is paradoxically overexpressed in several tumors not associated with HPV infection, however, is not clear. Atkins et al. noted in their discussion that overexpression of p16 in such cases remains enigmatic, but may be due to a mutated gene or nonfunctional protein [20]. In the present study, we examined the prevalence of p16 expression in leiomyoma, STUMP, and leiomyosarcoma tumors using IHC methods. The prevalence and p16 IHC scores were significantly higher in leiomyosarcoma tumors compared with leiomyoma or STUMP tumors. Bodner-Adler et al. reported p16 expression in $12 \%$ in leiomyomas and $57 \%$ and in leiomyosarcomas, and our results are consistent with their findings [19], confirming that p16 protein is a critical factor in leiomyosarcoma development and may be a useful diagnostic marker of leiomyosarcoma. In contrast, the clinical importance of the significant increase in p16 expression in STUMP cases remains unclear. Due to its rarity and difficulty of diagnosis, the clinical behavior and prognosis of STUMP remains controversial. O'Neill et al. reported that p16 expression is significantly higher in leiomyosarcomas compared with other tumor types, but only 1 of 4 STUMP tumors showed p16 overexpression, so there was no statistical difference in p16 expression between leiomyomas and STUMPs [20]. Bodner-Adler et al. reported that 5 of 19 cases had positive p16 staining, but all 5 cases had focal staining and a favorable clinical outcome [19]. Ünver et al. observed that only 1 of 3 STUMP cases was positive for p16 and this case did not have recurrence after 120 months of follow-up [30]. Conversely, Ip et al. investigated 16 STUMP cases and 5 showed focal p16 staining and 2 showed diffuse p16 staining [31]. In their report, the 2 patients with diffuse p16 staining developed recurrent disease and the 5 with focal p16 expression remained disease-free in the follow-up period [31]. Atkins et al. observed that three of eight STUMP cases developed metastatic disease and two of these tumors demonstrated diffuse and strong p16 positivity [20]. Based on these findings, they suggested that p16 expression might be a useful marker for predicting the prognosis in STUMP cases [20]. In our study, comprising 13 STUMP tumors, 5 patients (38.4\%) had p16 protein expression and p16 positivity was significantly different between leiomyomas and STUMP or STUMP and leiomyosarcomas. Of the five cases, one showed diffuse staining and the others showed focal staining, and all cases have had a favorable prognosis to date. Our findings conflict with those of their reports. This disagreement might be due to the small number of cases in all of the reports, including our study, and also probably due to the absence of uniformity in the diagnosis of STUMP. In our study, the patients with STUMP had not been followed up for a long period of time, so a longer follow-up is needed. Diffuse p16 expression is a potential helpful tool for predicting the behavior of problematic STUMPs. The physiologic role of p16 overexpression in leiomyosarcoma is still unclear. Therefore, we investigated the association between the degree of p16 expression and variable clinical parameters and prognosis in leiomyosarcoma. There is no established useful prognostic marker of leiomyosarcoma, and currently only the clinical stage is thought to be associated with the prognosis. Ünver et al. reported no significant association between p16 expression and clinical outcome in leiomyosarcoma [30]. In our study, p16 IHC scores were significantly higher in tumors of more advanced stages, higher mitotic activity, and recurrence. There was no relationship between p16 IHC scores and age, tumor size, extent of necrosis, or degree of myometrial invasion. These findings indicate that high p16 expression might be associated with the aggressiveness of the leiomyosarcoma. Factors affecting the prognosis of patients with leiomyosarcoma have not been established, excluding clinical stage. The significance of the prognostic value of p16 in leiomyosarcoma is not yet clear. Overexpression of p16 is reported to be associated with a poor prognosis in several malignant tumors [11] [22] [29] [32]. On the other hand, in some tumors, such as oropharyngeal, head and neck squamous cell carcinoma, and uterine cervical cancer, p16 overexpression is related to a better prognosis [33]-[35]. In these tumors, HPV infection is thought to be a major cause of carcinogenesis and extensive expression of p16 is thought to be a surrogate marker of active high-risk HPV infection. Although the link between high p16 expression and a favorable outcome is unclear, in these HPV-associated tumors, HPV-positive tumors tend to respond to radiation and chemotherapy or exhibit less distal or lymph node metastasis. One potential explanation is that in these HPVassociated tumors, increased p16 protein acts as a functionally intact tumor suppressor to maintain cellular homeostasis. In our study, p16 overexpression in leiomyosarcoma was associated with unfavorable outcomes, such as shorter disease-free and overall survival. In contrast to HPV-positive tumors, overexpressed p16 in leiomyosarcoma might be nonfunctional, resulting in resistance to therapeutic radiation or chemotherapy. Few data regarding the significance of p16 expression in the clinical outcome in leiomyosarcoma patients are available. Bodner-Adler et al. showed no positive correlation between p16 expression and clinical prognosis [19]. This finding differs from our findings. We do not know the clear cause of this discrepancy, but both studies comprised 
only small number of cases. Therefore, to confirm the clinical prognostic significance of p16 expression, further studies are needed, including a larger scale study with multivariate analysis. Elucidation of the mechanism of upstream regulation leading to 16 expression might facilitate the development of better therapeutic strategies that may improve the clinical course of leiomyosarcoma.

\section{Conclusion}

In conclusion, the use of p16 immunohisitochemistry is a useful marker in differential diagnosis in various uterine smooth muscle tumors. Moreover, p16 overexpression possibly plays an important role in tumor progression and we demonstrate that p16 may be a predictor of overall survival in patients with leiomyosarcoma tumors.

\section{Conflict of Interest Statement}

The author(s) indicate no potential conflicts of interest.

\section{References}

[1] Zagouri, F., Dimopoulos, A.M., Fotiou, S., Kouloulias, V. and Papadimitriou, C.A. (2009) Treatment of Early Uterine Sarcomas: Disentangling Adjuvant Modalities. World Journal of Surgical Oncology, 7, 38. http://dx.doi.org/10.1186/1477-7819-7-38

[2] Ip, P.P. and Cheung, A.N. (2011) Pathology of Uterine Leiomyosarcomas and Smooth Muscle Tumours of Uncertain Malignant Potential. Best Practice \& Research Clinical Obstetrics \& Gynaecology, 25, 691-704. http://dx.doi.org/10.1016/j.bpobgyn.2011.07.003

[3] Sabah, M., Cummins, R., Leader, M. and Kay, E. (2005) Leiomyosarcoma and Malignant Fibrous Histiocytoma Share Similar Allelic Imbalance Pattern at 9p. Virchows Archiv, 446, 51-58. http://dx.doi.org/10.1007/s00428-004-1190-2

[4] Mittal, K. and Demopoulos, R.I. (2001) MIB-1 (Ki-67), p53, Estrogen Receptor, and Progesterone Receptor Expression in Uterine Smooth Muscle Tumors. Human Pathology, 32, 984-987. http://dx.doi.org/10.1053/hupa.2001.27113

[5] Leiser, A.L., Anderson, S.E., Nonaka, D., Chuai, S., Olshen, A.B., Chi, D.S., et al. (2006) Apoptotic and Cell Cycle Regulatory Markers in Uterine Leiomyosarcoma. Gynecologic Oncology, 101, 86-91. http://dx.doi.org/10.1016/j.ygyno.2005.09.055

[6] Hewedi, I.H., Radwan, N.A. and Shash, L.S. (2012) Diagnostic Value of Progesterone Receptor and p53 Expression in Uterine Smooth Muscle Tumors. Diagnostic Pathology, 7, 1. http://dx.doi.org/10.1186/1746-1596-7-1

[7] Kamb, A., Gruis, N.A., Weaver-Feldhaus, J., Liu, Q., Harshman, K., Tavtigian, S.V., et al. (1994) A Cell Cycle Regulator Potentially Involved in Genesis of Many Tumor Types. Science, 264, 436-440.

http://dx.doi.org/10.1126/science.8153634

[8] Kim, W.Y. and Sharpless, N.E. (2006) The Regulation of INK4/ARF in Cancer and Aging. Cell, 127, 265-275. http://dx.doi.org/10.1016/j.cell.2006.10.003

[9] Kratzke, R.A., Greatens, T.M., Rubins, J.B., Maddaus, M.A., Niewoehner, D.E., Niehans, G.A., et al. (1996) Rb and p16INK4a Expression in Resected Non-Small Cell Lung Tumors. Cancer Research, 56, 3415-3420.

[10] Caldas, C., Hahn, S.A., da Costa, L.T., Redston, M.S., Schutte, M., Seymour, A.B., et al. (1994) Frequent Somatic Mutations and Homozygous Deletions of the p16 (MTS1) Gene in Pancreatic Adenocarcinoma. Nature Genetics, 8, 27-32. http://dx.doi.org/10.1038/ng0994-27

[11] Liu, T.J., Niu, Y., Feng, Y.M., Niu, R.F., Yu, Y., Lv, A., et al. (2008) Methylation of CpG Islands of p16 ${ }^{\mathrm{INK} 4 \mathrm{a}}$ and cyclinD1 Overexpression Associated with Progression of Intraductal Proliferative Lesions of the Breast. Human Pathology, 39, 1637-1646. http://dx.doi.org/10.1016/j.humpath.2008.04.001

[12] Asamoto, M., Iwahori, Y., Okamura, T., Shirai, T. and Tsuda, H. (1997) Decreased Expression of the p16/MTS1 Gene without Mutation Is Frequent in Human Urinary Bladder Carcinomas. Japanese Journal of Clinical Oncology, 27, 22-25. http://dx.doi.org/10.1093/jico/27.1.22

[13] Kawaguchi, K., Oda, Y., Saito, T., Yamamoto, H., Tamiya, S., Takahira, T., et al. (2003) Mechanisms of Inactivation of the p16 $6^{\mathrm{INK} 4 \mathrm{a}}$ Gene in Leiomyosarcoma of Soft Tissue: Decreased p16 Expression Correlates with Promoter Methylation and Poor Prognosis. The Journal of Pathology, 201, 487-495. http://dx.doi.org/10.1002/path.1419

[14] Surowiak, P., Materna, V., Maciejczyk, A., Pudelko, M., Suchocki, S., Kedzia, W., et al. (2008) Decreased Expression of p16 in Ovarian Cancers Represents an Unfavourable Prognostic Factor. Histology and Histopathology, 23, 531-538.

[15] Lesnikova, I., Lidang, M., Hamilton-Dutoit, S. and Koch, J. (2009) p16 as a Diagnostic Marker of Cervical Neoplasia: A Tissue Microarray Study of 796 Archival Specimens. Diagnostic Pathology, 4, 22. 
http://dx.doi.org/10.1186/1746-1596-4-22

[16] Branca, M., Ciotti, M., Santini, D., Di Bonito, L., Giorgi, C., Benedetto, A., et al. (2004) p16 ${ }^{\mathrm{INK} 4 \mathrm{~A}}$ Expression Is Related to Grade of CIN and High-Risk Human Papillomavirus but Does Not Predict Virus Clearance after Conization or Disease Outcome. International Journal of Gynecological Pathology, 23, 354-365. http://dx.doi.org/10.1097/01.pgp.0000139639.79105.40

[17] Reuschenbach, M., Vinokurova, S. and von Knebel Doeberitz, M. (2011) HPV-Associated Carcinomas of the Female Genital Tract. Molecular Mechanisms of Development. Der Pathologe, 32, 451-460. http://dx.doi.org/10.1007/s00292-011-1474-7

[18] Yemelyanova, A., Ji, H., Shih, I.M., Wang, T.L., Wu, L.S. and Ronnett, B.M. (2009) Utility of p16 Expression for Distinction of Uterine Serous Carcinomas from Endometrial Endometrioid and Endocervical Adenocarcinomas: Immunohistochemical Analysis of 201 Cases. American Journal of Surgical Pathology, 33, 1504-1514. http://dx.doi.org/10.1097/PAS.0b013e3181ac35f5

[19] Bodner-Adler, B., Bodner, K., Czerwenka, K., Kimberger, O., Leodolter, S. and Mayerhofer, K. (2005) Expression of p16 Protein in Patients with Uterine Smooth Muscle Tumors: An Immunohistochemical Analysis. Gynecologic Oncology, 96, 62-66. http://dx.doi.org/10.1016/j.ygyno.2004.09.026

[20] Atkins, K.A., Arronte, N., Darus, C.J. and Rice, L.W. (2008) The Use of p16 in Enhancing the Histologic Classification of Uterine Smooth Muscle Tumors. American Journal of Surgical Pathology, 32, 98-102. http://dx.doi.org/10.1097/PAS.0b013e3181574d1e

[21] O’Neill, C.J., McBride, H.A., Connolly, L.E. and McCluggage, W.G. (2007) Uterine Leiomyosarcomas Are Characterized by High p16, p53 and MIB1 Expression in Comparison with Usual Leiomyomas, Leiomyoma Variants and Smooth Muscle Tumours of Uncertain Malignant Potential. Histopathology, 50, 851-858. http://dx.doi.org/10.1111/j.1365-2559.2007.02699.x

[22] Zhao, W., Huang, C.C., Otterson, G.A., Leon, M.E., Tang, Y., Shilo, K., et al. (2012) Altered p16 ${ }^{\mathrm{INK} 4}$ and RB1 Expressions Are Associated with Poor Prognosis in Patients with Non-Small Cell Lung Cancer. Journal of Oncology, 2012, Article ID: 957437. http://dx.doi.org/10.1155/2012/957437

[23] Reed, J.A., Loganzo Jr., F., Shea, C.R., Walker, G.J., Flores, J.F., Glendening, J.M., et al. (1995) Loss of Expression of the p16/Cyclin-Dependent Kinase Inhibitor 2 Tumor Suppressor Gene in Melanocytic Lesions Correlates with Invasive Stage of Tumor Progression. Cancer Research, 55, 2713-2718.

[24] Bartsch, D., Shevlin, D.W., Callery, M.P., Norton, J.A., Wells Jr., S.A. and Goodfellow, P.J. (1996) Reduced Survival in Patients with Ductal Pancreatic Adenocarcinoma Associated with CDKN2 Mutation. Journal of the National Cancer Institute, 88, 680-682. http://dx.doi.org/10.1093/jnci/88.10.680

[25] Murai, Y., Hayashi, S., Takahashi, H., Tsuneyama, K. and Takano, Y. (2005) Correlation between DNA Alterations and p53 and p16 Protein Expression in Cancer Cell Lines. Pathology-Research and Practice, 201, 109-115. http://dx.doi.org/10.1016/j.prp.2005.01.002

[26] Wang, P., Pei, R.G., Lu, Z.M., Rao, X.S. and Liu, B.G. (2013) Methylation of p16 CpG Islands Correlated with Metastasis and Aggressiveness in Papillary Thyroid Carcinoma. Journal of the Chinese Medical Association, 76, 135-139. http://dx.doi.org/10.1016/j.jcma.2012.11.007

[27] Bartoletti, R., Cai, T., Nesi, G., Roberta Girardi, L., Baroni, G. and Dal Canto, M. (2007) Loss of P16 Expression and Chromosome 9p21 LOH in Predicting Outcome of Patients Affected by Superficial Bladder Cancer. Journal of Surgical Research, 143, 422-427. http://dx.doi.org/10.1016/j.jss.2007.01.012

[28] Duncan, L.D., Winkler, M., Carlson, E.R., Heidel, R.E., Kang, E. and Webb, D. (2013) p16 Immunohistochemistry Can Be Used to Detect Human Papillomavirus in Oral Cavity Squamous Cell Carcinoma. Journal of Oral and Maxillofacial Surgery, 71, 1367-1375. http://dx.doi.org/10.1016/j.joms.2013.02.019

[29] Milde-Langosch, K., Bamberger, A.M., Rieck, G., Kelp, B. and Loning, T. (2001) Overexpression of the p16 Cell Cycle Inhibitor in Breast Cancer Is Associated with a More Malignant Phenotype. Breast Cancer Research and Treatment, 67, 61-70. http://dx.doi.org/10.1023/A:1010623308275

[30] Ünver, N.U., Acikalin, M.F., Öner, Ü., Ciftci, E., Ozalp, S.S. and Colak, E. (2011) Differential Expression of P16 and P21 in Benign and Malignant Uterine Smooth Muscle Tumors. Archives of Gynecology and Obstetrics, 284, 483-490. http://dx.doi.org/10.1007/s00404-010-1690-Z

[31] Ip, P.P., Cheung, A.N. and Clement, P.B. (2009) Uterine Smooth Muscle Tumors of Uncertain Malignant Potential (STUMP): A Clinicopathologic Analysis of 16 Cases. American Journal of Surgical Pathology, 33, 992-1005. http://dx.doi.org/10.1097/PAS.0b013e3181a02d1c

[32] Huang, L.W. and Lee, C.C. (2012) P16 ${ }^{\mathrm{INK} 4 \mathrm{~A}}$ Overexpression Predicts Lymph Node Metastasis in Cervical Carcinomas. Journal of Clinical Pathology, 65, 117-121. http://dx.doi.org/10.1136/jclinpath-2011-200362

[33] Rischin, D., Young, R.J., Fisher, R., Fox, S.B., Le, Q.T., Peters, L.J., et al. (2010) Prognostic Significance of p16 ${ }^{\mathrm{INK} 4 \mathrm{~A}}$ 
and Human Papillomavirus in Patients with Oropharyngeal Cancer Treated on TROG 02.02 Phase III Trial. Journal of Clinical Oncology, 28, 4142-4148. http://dx.doi.org/10.1200/JCO.2010.29.2904

[34] Lau, H.Y., Brar, S., Klimowicz, A.C., Petrillo, S.K., Hao, D., Brockton, N.T., et al. (2011) Prognostic Significance of p16 in Locally Advanced Squamous Cell Carcinoma of the Head and Neck Treated with Concurrent Cisplatin and Radiotherapy. Head \& Neck, 33, 251-256. http://dx.doi.org/10.1002/hed.21439

[35] Schwarz, J.K., Lewis Jr., J.S., Pfeifer, J., Huettner, P. and Grigsby, P. (2012) Prognostic Significance of p16 Expression in Advanced Cervical Cancer Treated with Definitive Radiotherapy. International Journal of Radiation Oncology, Biology, Physics, 84, 153-157. http://dx.doi.org/10.1016/j.ijrobp.2011.11.032 